\title{
Transformation of B cell lymphoma to histiocytic sarcoma: somatic mutations of PAX-5 gene with loss of expression cannot explain transdifferentiation
}

\author{
Assia Bassarova • Gunhild Trøen - Alexander Fosså • \\ Ida Munster Ikonomou • Klaus Beiske • \\ Jahn M. Nesland • Jan Delabie
}

Received: 3 March 2009 / Accepted: 22 April 2009/Published online: 9 May 2009

(C) Springer-Verlag 2009

\begin{abstract}
Transdifferentiation of B cell lymphoma of germinal center cell origin to histiocytic sarcoma has recently been described but is a rare occurrence. The cause for loss of $\mathrm{B}$ cell differentiation in these lymphomas is unknown. We investigated whether somatic hypermutation of the $P A X-5$ gene, a transcription factor that is important for maintaining B cell identity and is frequently mutated in $\mathrm{B}$ cell lymphomas of germinal center cell origin, might be a cause for loss of PAX-5 expression and thus B cell phenotype. However, no somatic hypermutation of the $P A X-5$ gene was detected in the two cases we studied. The molecular basis for transdifferentiation of B cell lymphoma to histiocytic sarcoma remains therefore unresolved.
\end{abstract}

Keywords PAX-5 - B-cell · Lymphoma .

Histiocytic sarcoma $\cdot$ Transdifferentiation

\section{Introduction}

Phagocytes are derived from monocytes that enter the blood and migrate into tissues to differentiate into macrophages/ histiocytes [1,2]. Tumors of histiocytes and dendritic cells are extremely rare and their classification is still controver-

A. Bassarova $(\bowtie) \cdot$ G. Trøen $\cdot$ I. M. Ikonomou $\cdot$ K. Beiske

J. M. Nesland $\cdot$ J. Delabie

Department of Pathology, Oslo University Hospital-

The Norwegian Radium Hospital, University of Oslo, Montebello 0310 Oslo, Norway

e-mail: assia.bassarova@radiumhospitalet.no

\section{A. Fosså}

Department of Oncology, Oslo University HospitalThe Norwegian Radium Hospital, University of Oslo, Oslo, Norway sial $[1,2]$. In the last World Health Organization classification [1], primary histiocytic sarcoma (HS) is defined as a tumor with morphologic and immunohistochemical features similar to those of mature histiocytes and usually lack clonal immunoglobulin and $\mathrm{T}$ cell receptor genes. Of interest, those tumors frequently occur in patients with another malignant tumor. For example, a subset of cases with HS occurs in patients with previously diagnosed mediastinal germ cell tumors; other cases are associated with malignant lymphomas [3-6].

Of interest is one recent publication which described rare cases of HS occurring as a transformed tumor from B cell lymphoma [7]. Here, we report two such cases and set out to look for a mechanism explaining this transdifferentiation.

Somatic hypermutation affects rearranged immunoglobulin genes in B cells and results in higher affinity of the antibody. However, aberrant somatic hypermutation that affects other genes, including the $P A X-5$ gene, has been described in B cell lymphomas [8]. PAX-5 is a transcription factor that is important for maintaining B cell identity [9]. We therefore investigated whether $P A X-5$ aberrant somatic hypermutation was a cause of the lack of PAX-5 expression and a probable cause of transdifferentiation.

\section{Materials and methods}

Clinical histories

Patient 1 A 53-year-old male patient was diagnosed in 1995 with stage IVA follicular lymphoma, grade 2 with generalized lymph node and bone marrow involvement. He was treated with chlorambucil/prednisolone for 6 months. A first relapse was detected 7 years later and the patient was treated again. 
Table 1 List of the antibodies used for immunohistochemistry

Fig. 1 a, b Lymph node with follicular lymphoma; c, d, e neck lesion with histiocytic sarcoma; $\mathbf{f}$ bone marrow infiltration with diffuse large B cell lymphoma (patient 2; H\&E)

\begin{tabular}{llll}
\hline Antibody (clone) & Source & Dilution & HIER \\
\hline CD45 (LCA, 2B11/PD7/26) & DAKO Cytomation, Denmark A/S & $1: 100$ & LpH \\
CD20 (L-26) & DAKO Cytomation, Denmark A/S & $1: 300$ & LpH \\
CD3 (SP7, rabbit monoclonal) & LabVision Corporation, UK & $1: 150$ & LpH \\
CD79a (SP-18, rabbit monoclonal) & LabVision Corporation, UK & $1: 400$ & T/E \\
PAX-5 (24) & BD Transduction Laboratories ${ }^{\mathrm{TM}}$ & $1: 40$ & $\mathrm{~T} / \mathrm{E}$ \\
CD10 (56C6) & Novocastra ${ }^{\mathrm{TM}}$ & $1: 40$ & $\mathrm{~T} / \mathrm{E}$ \\
BCL-6 (PG-B6p) & DAKO Cytomation, Denmark A/S & $1: 40$ & $\mathrm{~T} / \mathrm{E}$ \\
BCL-2 (Bcl-2/100/D5) & Novocastra ${ }^{\mathrm{TM}}$ & $1: 20$ & $\mathrm{~T} / \mathrm{E}$ \\
CD21 (2G9) & Novocastra & $1: 20$ & $\mathrm{~T} / \mathrm{E}$ \\
CD68 (PG-M1) & DAKO Cytomation, Denmark A/S & $1: 250$ & $\mathrm{~T} / \mathrm{E}$ \\
CD14 (7) & Novocastra ${ }^{\mathrm{TM}}$ & $1: 160$ & $\mathrm{~T} / \mathrm{E}$ \\
CD1a (MTB1) & Novocastra & $1: 5$ & $\mathrm{~T} / \mathrm{E}$ \\
PU1 (polyclonal) & Santa Cruz Biotechnology & $1: 300$ & $\mathrm{~T} / \mathrm{E}$ \\
MAF-B (goat polyclonal) & Santa Cruz Biotechnology & $1: 2000$ & $\mathrm{~T} / \mathrm{E}$ \\
S-100 (polyclonal) & DAKO Cytomation, Denmark A/S & $1: 1500$ & $\mathrm{LpH}$ \\
Ki67 (MIB-1) & DAKO Cytomation, Denmark A/S & $1: 150$ & $\mathrm{~T} / \mathrm{E}$ \\
\hline
\end{tabular}
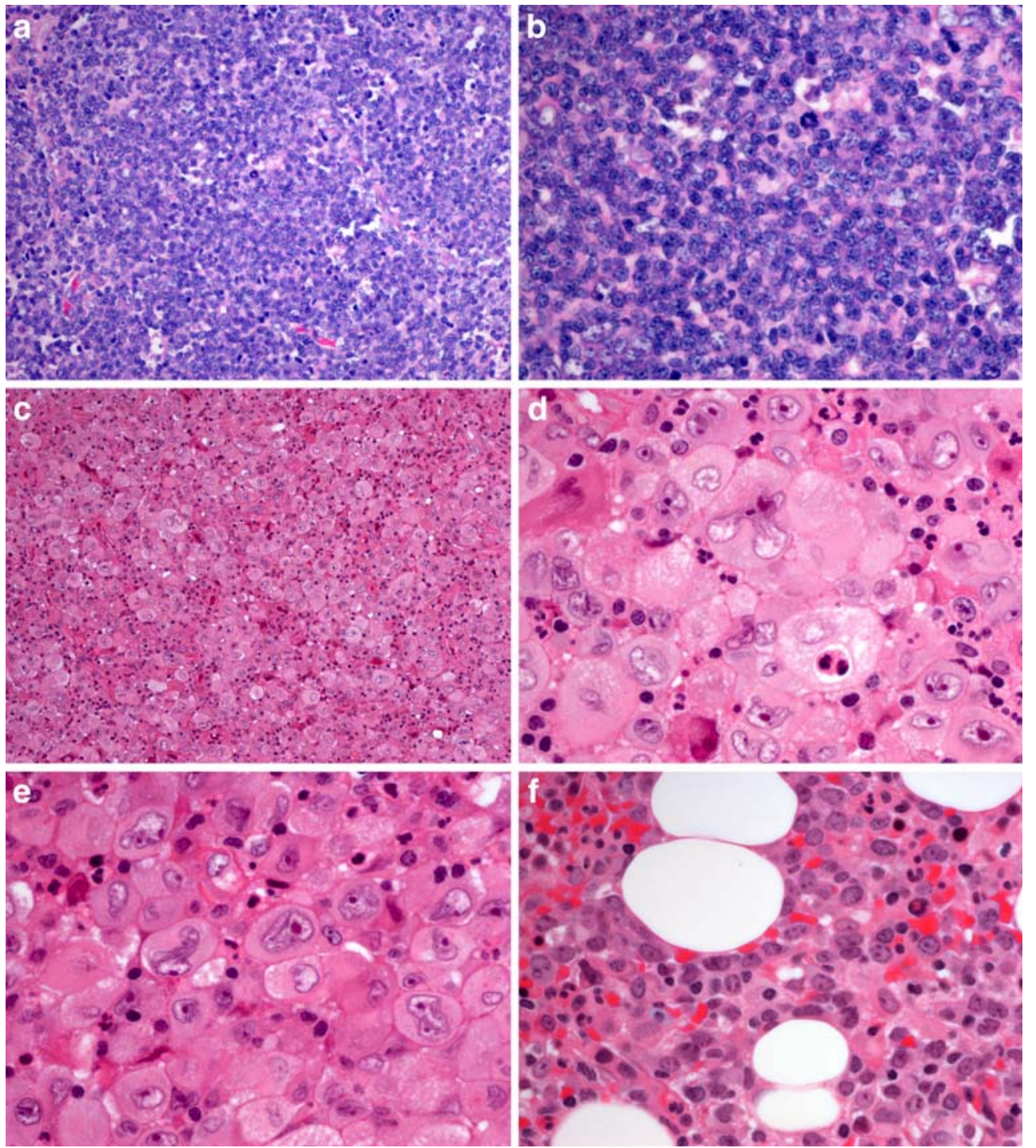


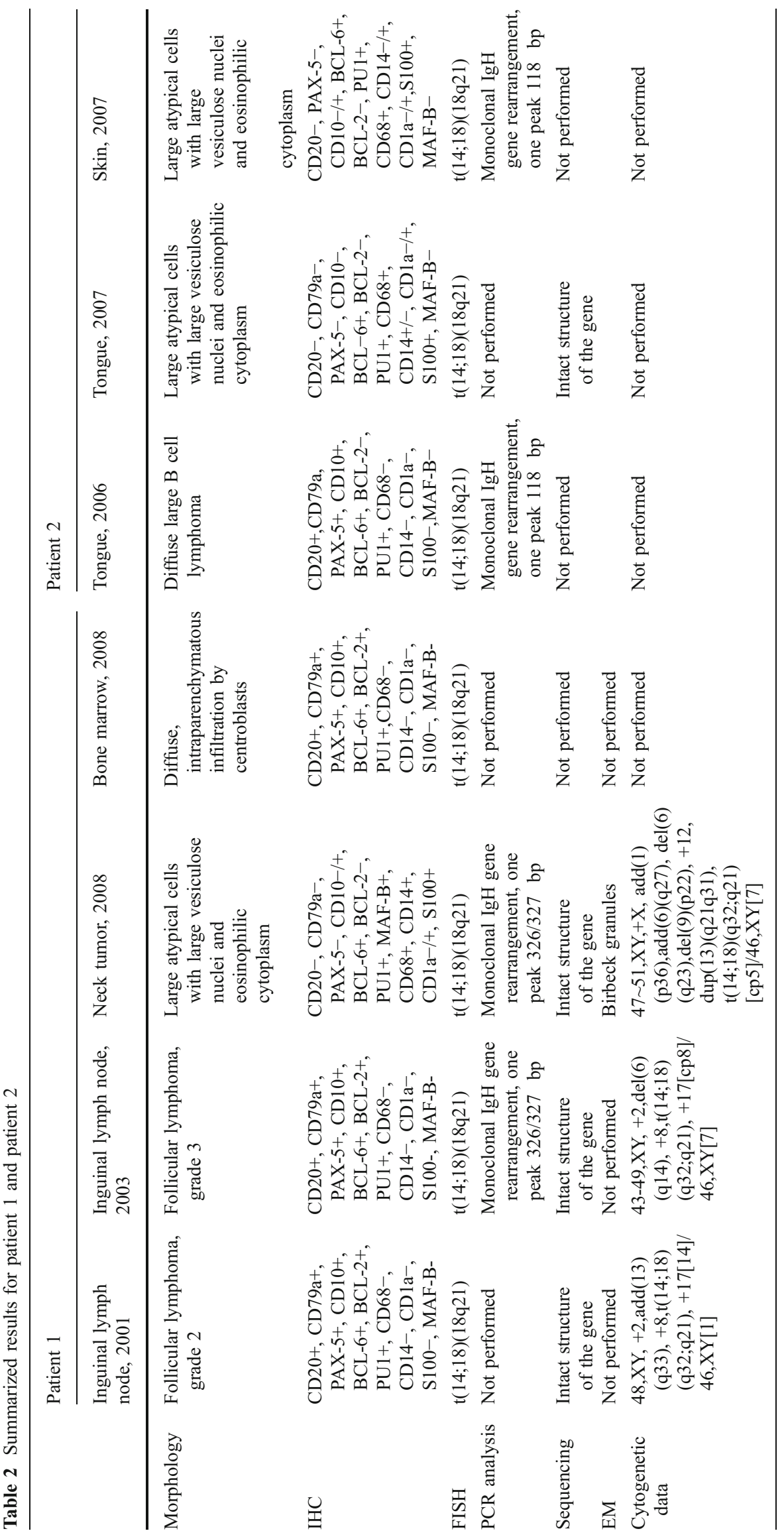


One year later, a second recurrence with grade 3B FL was diagnosed. He received six courses of R-CHOP. A third relapse with slow-growing lymph nodes and bone marrow involvement was documented 3 years later and Zevalin was administered. Thirteen years after the initial diagnosis, the patient developed a quickly growing tumor on the left side of the neck. The biopsy showed a diffuse infiltration with large atypical cells with large irregular indented nuclei and eosinophilic cytoplasm with the immunophenotype of histiocytes. The bone marrow showed infiltration by diffuse large B cell lymphoma (DLBCL). The patient was treated with five courses of DHAP, with a rapid response of the cervical tumor. The DLBCL in the bone marrow progressed rapidly and the patient died shortly after the last DHAP course.
Fig. 2 a, b Lymph node with follicular lymphoma (CD20 and bcl-2, IHC); c, d, e histiocytic sarcoma (CD14, MAF-B, and $\mathrm{CD} 1 \mathrm{a}, \mathrm{IHC}) ; \mathbf{f}$ bone marrow infiltration with diffuse large B cell lymphoma (PAX-5, IHC); $\mathbf{g}$ PCR analysis of follicular lymphoma and histiocytic sarcoma; h electron microscopy - detail of Birbeck granula (patient 2)
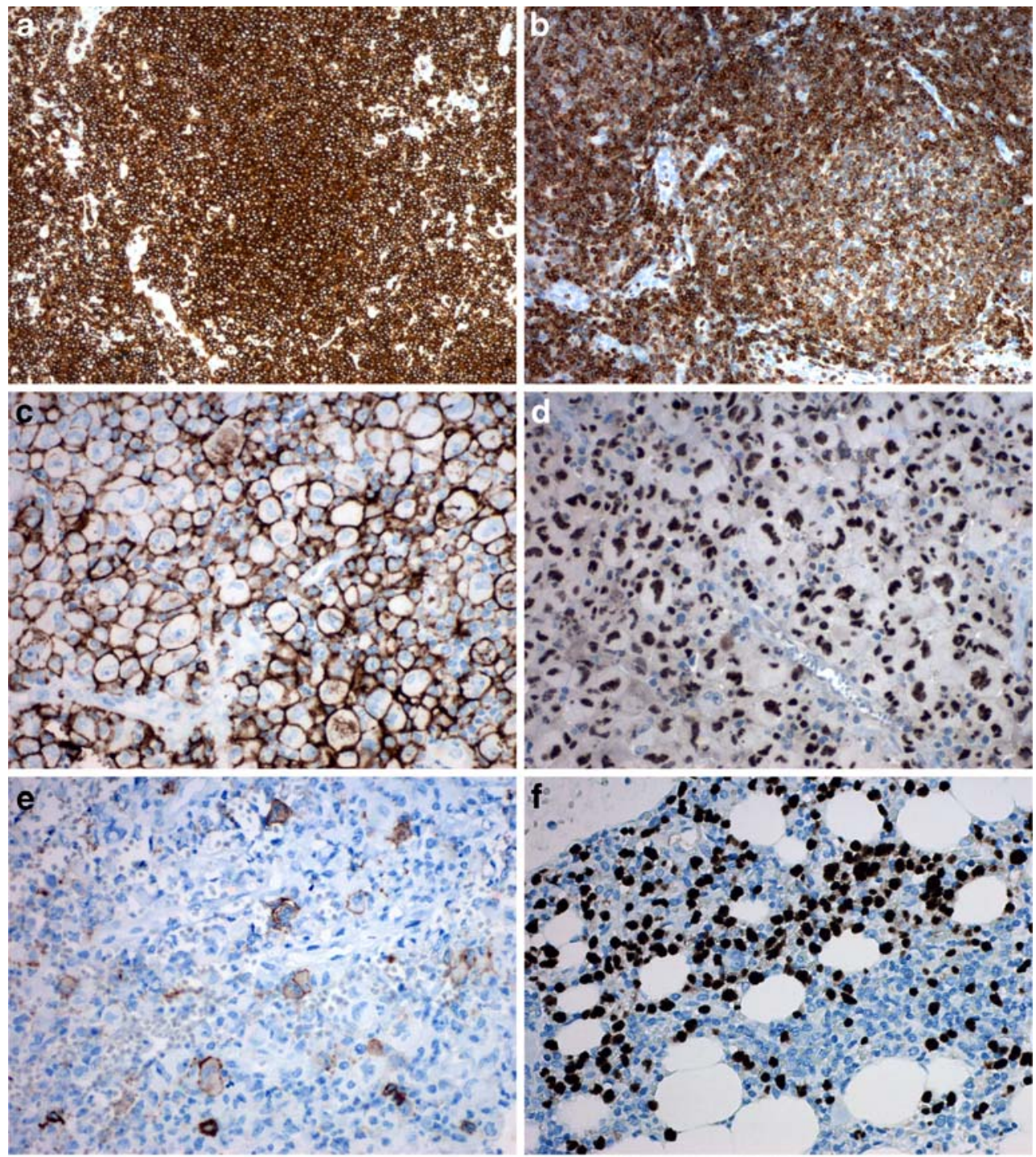

\section{g}

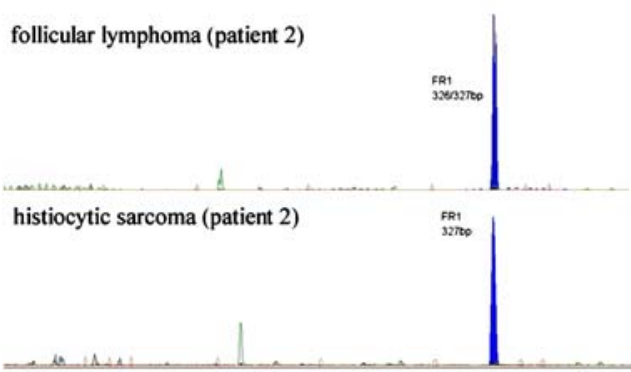


Patient 2 A 62-year-old male patient presented with nodular lesion on the tongue and enlarged cervical lymph nodes and was diagnosed as diffuse large cell B cell lymphoma stage IIA. He received eight courses of R-CHOP-14 with complete remission. One year later, a local relapse with large pleomorphic cells and with the immunophenotype of histiocytic cells was diagnosed. The tumor invaded the base of the tongue, hypopharynx, and cervical lymph nodes. Complete remission after four courses of MIME was achieved and involved field radiotherapy with 40 Gy was given. A second recurrence developed shortly thereafter with similar lesions on the abdomen. The patient received no further treatment and died of tumor progression.

Immunohistochemistry The paraffin blocks were cut at 4$6 \mu \mathrm{m}$, dried overnight at $60{ }^{\circ} \mathrm{C}$, and dewaxed in xylene. A standard panel with 16 primary antibodies was used in both cases (Table 1). Visualization was performed using the EnVision ${ }^{\circledR}$ detection system (DAKO Cytomation, Denmark A/S) according to the manufacturer's instructions. Appropriate positive and negative controls were used.

Fluorescent in situ hybridization (FISH) analysis Fluorescent in situ hybridization analysis was performed on formalin-fixed tissue specimens, using a BCL-2 split signal DNA probe (Y5407, DAKO Cytomation, Denmark A/S). The slides were deparaffinized, immersed in pretreatment buffer (K5599, DAKO Cytomation, Denmark A/S) for $10 \mathrm{~min}$ at $95^{\circ} \mathrm{C}$, followed by pepsin pretreatment, and dehydrated in ethanol. After a denaturation step for $10 \mathrm{~min}$ at $80^{\circ} \mathrm{C}$, hybridization was performed at $37^{\circ} \mathrm{C}$ overnight. The next day, the slides were postwashed in saline sodium citrate (SSC) buffer, counterstained with 4',6-diamidino-2phenylindole, and evaluated using fluorescent microscope Olympus BX61.

Polymerase chain reaction (PCR) analysis and sequencing DNA was extracted from fresh snap-frozen tissue or paraffin-embedded tissue using QIAmp tissue kit (Qiagen, Hilden, Germany). Analysis of immunoglobulin heavy chain $(\operatorname{IgH})$ gene rearrangement was done using $\operatorname{IgH}$ variable gene $(\mathrm{VH})$ family-specific primers (VH1 trough VH6) and VH consensus primers covering the VH framework regions (FR) together with the consensus joining region gene primers as described in the BIOMED-2 protocol [10]. For one sample (case 2, DLBCL), an FR-3specific primer was used due to the fact that only paraffinembedded tissue was available. IgH polymerase chain reaction sequences were directly sequenced by using the Big Dye terminator sequencing kit and the Genetic Analyzer 3100 (Applied Biosystems, Weiterstadt, Germany). The sequences obtained were analyzed by comparison with the $\mathrm{V}$ base database (www.vbase2.org).

As somatic hypermutation affects only the $5^{\prime}$ region of affected genes, analysis of the $5^{\prime}$ end of the PAX5 gene was performed using primer pairs covering exon 1 (1A and $1 \mathrm{~B})$; exons 2 and 3 were used as previously described [11].

\section{Electron microscopy}

Formalin-fixed paraffin-embedded material was used. The paraffin block was cut and the section was immersed in xylene and then rehydrated in alcohol. Then, the material was

\section{a Case 1:}

FL

humIGHV173

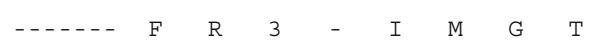

CDR3 - IMGT

TATTTACAAATGAACAGTTTGAAAACTGAGGACACGGCCTCCTATTACTGTACAAGAGCGGACTGTATTGCTGGTGCTTGTTATTTTGACTCT

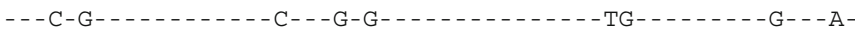

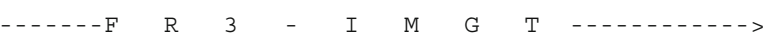
CDR3 - IMGT

Histiocytic_sarcoma humIGHV173

b Case 2:
TATTTGCAATTGAACAGTTTGAAAATTGAGGACACGGCCTCCTATTATTGTACAAGAGCGGACTGTATTGCTGGTGTTTGTTATTTTGACTCT

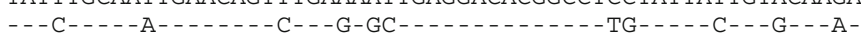

DLBCL

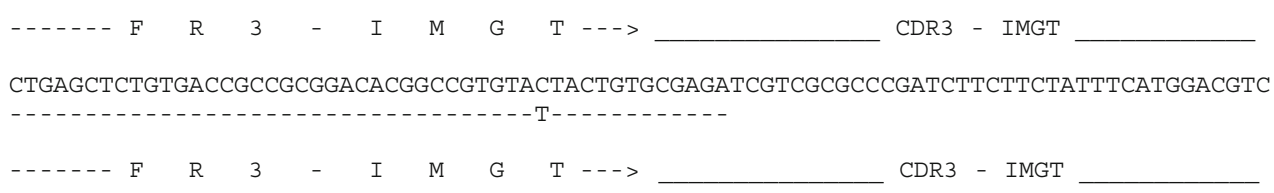

Histiocytic sarcoma humIGHV117

CTAAACTCTGTGACCGCTGCGGACACGGCCGTGTACTACTGTGCGAGATCGTCGCGCCCGATCTTCTTCTATTTCATGGACGTC

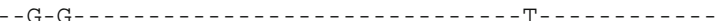

Fig. 3 Sequences of IgH gene rearrangements. The FR3 and CDR3 region sequences are arranged with the identified patient sample at the top, compared with the lower $\mathrm{V}$ base gene bank sequence with closest homology. Dashed lines indicate that the sequence is identical to the gene bank sequence, and a nucleotide indicates difference. a Patient 1, follicular lymphoma compared with histiocytic sarcoma. b Patient 2, diffuse large B cell lymphoma compared with histiocytic sarcoma 
immersed in cacodylate buffer for $24 \mathrm{~h}$ and additional postfixation in osmium tetraoxyde for $1 \mathrm{~h}$ was performed. After $1 \mathrm{~h}$ in uranyl acetate, the tissue was dehydrated, embedded in EPON; ultrathin sections were cut and evaluation was performed using Philips CM10 electron microscope.

\section{Cytogenetics}

Fresh tissue samples from patient 1 (two lymph node biopsies and a biopsy from a neck tumor) were manually minced and enzymatically treated until a suitable suspension of cells and cell clumps was obtained. After 5-7 days of culturing, colchicine was added for the last $4 \mathrm{~h}$ and the short-term cultures were harvested according to standard protocols. The chromosomes of the dividing cells were then G-banded and a karyotype was established in accordance with the recommendations of the International System for Human Cytogenetic Nomenclature.

\section{Results and discussion}

In both patients, $\mathrm{B}$ cell lymphoma with a germinal center cell phenotype and $t(14 ; 18)$ at first presentation was diagnosed. A diagnosis of low-grade follicular lymphoma with a typical morphology and immunophenotype was established on the first biopsy in patient 1 (Fig. 1, Table 2). Patient 2 presented with diffuse large B cell lymphoma with germinal center cell phenotype. In both patients, the initial lymphomas expressed CD20, PAX-5, CD10, and BCL-6 but no histiocytic markers (Fig. 2, Table 2). The recurrence in both patients showed a different histology with large pleomorphic cells with abundant eosinophilic cytoplasm and large irregular nuclei with focal nuclear grooves (Fig. 1, Table 2). The immunophenotype of these recurrences was also different; notably, B cell markers were lost and a strong expression of S100, MAF-B, CD68, CD14, and focal expression of CD1a was documented (Fig. 2, Table 2). Epstein Barr virus-encoded RNA in situ hybridization was negative in both patients. No clinical or morphological evidence of hemophagocytic syndrome was noted in the two patients. Electron microscopy was only successful in patient 2 and showed Birbeck granules (Fig. 2). We found a clonal relationship between the tumors with different phenotypes in both patients as documented by similarly rearranged $\operatorname{IgH}$ gene sequences, respectively (Figs. 2 and 3).

Since PAX-5 is crucial for maintaining B cell identity and since the transformed tumors in both patients had lost PAX-5 expression, we sequenced the $5^{\prime}$ part of the PAX-5 gene to detect probable aberrant somatic hypermutations [9]. Aberrant somatic hypermutation is known to occur in B cell lymphoma and can affect the expression of target genes
$[8,12]$. However, sequence analysis of the $5^{\prime}$ part of $P A X-5$, including exons 1,2 , and 3 of the $P A X-5$ gene, shows an intact structure of the gene without somatic hypermutation. Therefore, aberrant somatic hypermutation of $P A X-5$ is an unlikely cause of loss of expression and transdifferentiation at least in our two patients. We have not investigated whether deletion of $P A X-5$ gene might explain loss of PAX5 expression in our two cases. However, this hypothesis seems to be unlikely in view of the fact that the $P A X-5$ gene could easily be amplified from DNA extracted from the tumors. Morphological control of the tissues used for DNA extraction assured that the DNA extracts were representative of the tumor and confirmed that a majority of tumor cells were present.

Of interest, cytogenetic data were obtained in patient 1 where the follicular lymphoma as well as the histiocytic sarcoma showed $\mathrm{t}(14 ; 18)(18 \mathrm{q} 21)$. In addition, the recurrent histiocytic sarcoma showed a more complex karyotype with multiple deletions, duplications, and insertions. The latter suggests that chromosomal instability is at least part of the transformation process.

In conclusion, it is of interest that mature neoplastic B cells may display translineage transformation. This seems hitherto restricted to B cell lymphomas of germinal center cell origin with $\mathrm{t}(14 ; 18)$. The mechanisms by which that occur are yet not clear, but aberrant somatic hypermutation of the $P A X-5$ gene seems to be an unlikely cause.

Conflict of interest The authors declare that they have no conflict of interest.

\section{References}

1. Jaffe R, Pileri S, Facchetti F, Jones DM, Jaffe ES (2008) Histiocytic and dendritic neoplasms. In: Swerdlow S et al (eds) World Health Organization classification of tumors. Tumours of haematopoietic and lymphoid tissues, 4th edn. IARC, Lyon, p 354

2. Pileri SA, Grogan TM, Harris NL et al (2002) Tumours of histiocytes and accessory dendritic cells: an immunohistochemical approach to classification from the International Lymphoma Study Group based on 61 cases. Histopathology 41:1-29

3. Nichols CR, Roth BJ, Heerema N et al (1990) Hematologic neoplasia associated with primary mediastinal germ-cell tumors. N Engl J Med 322:1425-1429

4. Baer MR, Krantz SB, Cousar JB et al (1986) Malignant histiocytoses developing in patients with B-cell lymphomas. Report of two cases. Cancer 57:2175-2184

5. Wetzler M, Kurzrock R, Goodacre AM et al (1995) Transformation of chronic lymphocytic leukemia to lymphoma of true histiocytic type. Cancer 76:609-617

6. Vasef MA, Zaatari GS, Chan WC et al (1995) Dendritic cell tumors associated with low-grade B-cell malignancies. Report of three cases. Am J Clin Pathol 104:696-701

7. Feldman AL, Arber DA, Pittaluga S et al (2008) Clonally related follicular lymphomas and histiocytic/dendritic cell sarcomas: 
evidence for transdifferentiation of the follicular lymphoma clone. Blood 111:5433-5439

8. Pasqualucci L, Neumeister P, Goossens T et al (2001) Hypermutation of multiple proto-oncogenes in B-cell diffuse large-cell lymphomas. Nature 412:341-346

9. Nutt SL, Heavey B, Rolink AG, Busslinger M (1999) Commitment to the B-lymphoid lineage depends on the transcription factor Pax5. Nature 401:556-562

10. van Dongen JJ, Langerak AW, Brüggemann M et al (2003) Design and standardization of PCR primers and protocols for detection of clonal immunoglobulin and T-cell receptor gene recombinations in suspect lymphoproliferations: report of the BIOMED-2 Concerted Action BMH4-CT98-3936. Leukemia $17: 2257-2317$

11. Mullighan GC, Goorha S, Radtke I et al (2007) Genome-wide analysis of genetic alterations in acute lymphoblastic leukaemia. Nature 446:758-764

12. Halldorsdottir AM, Fruhwirth M, Deutsch A et al (2008) Quantifying the role of aberrant somatic hypermutation in transformation of follicular lymphoma. Leuk Res 32:1015-1021 